we analyzed data from the study visit with higher disease activity from each patient, applying multivariate linear regression analysis, with PGA as dependent variable/gold-standard. Independent variables tested in the models included items from SLEDAI-2K and continuous variables for swollen joint count, proteinuria, platelet and white blood cells counts. Some features absent from SLEDAI, such as hemolytic anemia, gastrointestinal and cardiopulmonary involvement were added to the model.

To assess correlation validity we performed a Spearman's correlation between the SLE-DAS, PGA and SLEDAI-2K at last follow-up visit. We tested performance of SLEDAI-2K (change $\geq 4$ ) and SLE-DAS to discriminate a clinically meaningful worsening and improvement in SLE disease activity (change in PGA $\geq 0.3$ ) using Receiver Operating Characteristic (ROC) curve analysis. We determined the best cut-offs values of SLE-DAS to detect changes in PGA $>0.3$ and calculated the sensitivity, specificity, positive and negative predictive values (PPV, NPV). Statistical significance was set at 0.05 .

Results: The final SLE-DAS model included 17 items. The SLE-DAS score at last follow-up visit presented high correlation with PGA (rho $=0.975, p<0.0005$ ) and SLEDAI-2K (rho=0.94, $\mathrm{p}<0.0005$ ). For improvement in $P G A \geq 0.3$, in $R O C$ analysis a change in SLE-DAS presented a much higher performance [area under curve $(\mathrm{AUC})=0.927 \quad(95 \% \quad \mathrm{Cl}=0.885-0.969, \quad \mathrm{p}<0.0005)]$ than SLEDAI-2K [AUC=0.787 (95\% Cl=0.718-0.857), $\mathrm{p}<0.0005$ ] (figure 1). For worsening of $P G A \geq 0.3$, change in SLE-DAS and SLEDAI-2K presented an AUC of 0.994 (95\% $\mathrm{Cl}=0.988-1.000, \mathrm{p}<0.0005)$ and $0.914(95 \% \mathrm{Cl}=0.870-0.959, \mathrm{p}<0.0005)$, respectively (figure 1). The optimal discriminative cut-off for either a PGA increase or reduction was change in SLE-DAS $\geq 1.72$ (table 1 ).

Table 1 Performance of SLE-DAS and SLEDAI-2K to detect change in SLE disease activity

\begin{tabular}{lllllllll}
\hline & \multicolumn{3}{l}{$\Delta$ SLE-DAS $\geq \mathbf{1 . 7 2}$} & \multicolumn{5}{c}{$\Delta$ SLEDAI-2K $\geq \mathbf{4}$} \\
\cline { 2 - 8 } & Sens & Spec & PPV & NPV & Sens & Spec & PPV & NPV \\
\hline $\begin{array}{l}\text { Improvement } \\
\text { PGA } \geq-\mathbf{0 . 3}\end{array}$ & 82.1 & 96.9 & 87.3 & 95.4 & 44.8 & 96.5 & 76.9 & 87.0 \\
$\begin{array}{l}\text { Worsening PGA } \geq \\
+0.3\end{array}$ & 93.1 & 97.7 & 90.0 & 98.5 & 46.6 & 99.6 & 96.4 & 89.5 \\
\hline
\end{tabular}

Sens: Sensitivity(\%); Spec: Specificity(\%); PPV: Positive predictive value(\%); NPV: Non
predictive value(\%)
A

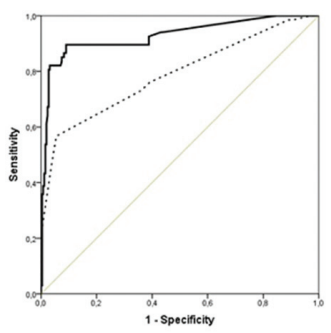

B

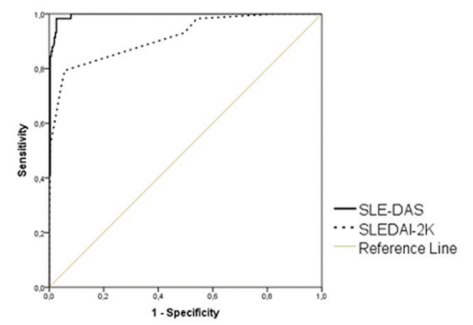

Figure 1 Receiver operating curve (ROC) comparing the performance of SLE-DAS and SLEDAI-2K to detect a clinical meaningful improvement (A) and worsening (B) in SLE disease activity.

Conclusions: The SLE-DAS presents good construct validity and much higher discriminative power to detect changes in SLE disease activity as compared to SLEDAI-2K. External validation in another SLE cohort is underway.

Disclosure of Interest: None declared

DOI: 10.1136/annrheumdis-2018-eular.3161

\section{FRI0642 SEPTIC ARTHRITIS SCREENING WITH A FAST DIAGNOSTIC TOOL USING MID INFRARED SPECTROSCOPY: A MULTI-CENTRIC STUDY}

J.-D. Albert ${ }^{1}$, M. Le Corvec ${ }^{2}$, A. Martin ${ }^{3}$, X. Guennoc ${ }^{3}$, C. David ${ }^{4}$, S. Hoang ${ }^{4}$, C. Guedes ${ }^{4}$, M. Ferreyra ${ }^{4}$, E. Hoppe ${ }^{5}$, B. Legoff' ${ }^{\text {, S. Jousse-Joulin' }}{ }^{7}$, H. Tariel ${ }^{2}$, O. Sire ${ }^{8}$, A. Jolivet-Gougeon ${ }^{9}$, P. Guggenbuhl' 1 , O. Loreal ${ }^{10}$. ${ }^{1}$ Rheumatology, $\mathrm{CHU}$ de Rennes, ${ }^{2}$ DIAFIR, RENNES, ${ }^{3}$ Rheumatology, CH Saint-Brieuc, Saint-Brieuc, ${ }^{4}$ Rheumatology, $\mathrm{CH}$ de Bretagne Atlantique, Vannes, ${ }^{5}$ Rheumatology, $\mathrm{CHU}$ Angers, Angers, ${ }^{6}$ Rheumatology, CHU Nantes, Nantes, ${ }^{7}$ Rheumatology, $\mathrm{CHU}$ de Brest, Brest, ${ }^{8}$ IRDL, FRE CNRS 3744, Vannes, ${ }^{9}$ Microbiology, CHU de Rennes, ${ }^{10}$ Institut Numecan, INSERM U1241, Rennes, France

Background: Septic arthritis diagnostic is an emergency which implies a treatment with antibiotics and hospitalization. The diagnosis is based on the cytobacteriological examination of the synovial fluid (SF), but direct bacteriological examination is insensitive and the result of the culture is obtained only after several days. Therefore, there is still a need for a rapid, simple and reliable method for the positive diagnosis of septic arthritis. Such method must allow avoiding both unrecognized septic arthritis leading to major functional consequences, and overdiagnosis that will induce unnecessary expensive hospitalization and unjustified treatment with consequences in term of health and social costs.

Mid-infrared (MIR) spectroscopy, that gives a metabolic profiling of biological samples, has been proposed for early and fast diagnosis.

Objectives: The objective of this study was to confirm ${ }^{(1)}$ the interest of mid-infrared (MIR) spectroscopy to discriminate synovial fluid samples from patients with septic arthritis from other causes of joint effusion.

Methods: Synovial fluids from patients referred for suspected arthropathies were prospectively collected in six hospitals in western France and stored at $80^{\circ} \mathrm{C}$. The infrared absorption spectrum was acquired for each of the frozen sam ples using a chalcogenide fibre sensor. The most informative spectral variables (allowing to discriminate between septic arthritis and non-septic arthritis with reference to cytobiological examination) were selected and then used to develop an algorithm. Non-frozen synovial fluids were also analysed at Rennes University Hospital, the pilot centre, to validate the algorithm.

Results: The cohort consists of synovial fluid samples from patients exhibiting various etiologies. These samples $(n=402)$, by using SF bacteriological analysis and culture and 16S PCR analysis were classified as septic arthritis $(n=30)$ or non septic arthritis $(n=372)$.

On the frozen samples the performances of the algorithm show a sensitivity of $97 \%$, a specificity of $71 \%$, a VPN of $99 \%$ and a VPP of $21 \%$, the area under the ROC curve (AUROC) was 0.91 .

Conclusions: This study confirms the interest of optical fibre infrared spectroscopy for the discrimination between septic and non septic synovial fluids. The high negative predictive value and the very short time (about ten minutes) required to obtain the result makes it possible to quickly rule out an infection diagnosis which could make it possible to avoid unnecessary hospitalization and antibiotic therapy.

\section{REFERENCE:}

1. Albert J-D, et al. Joint Bone Spine 2016;83:318-323.

Disclosure of Interest: J.-D. ALBERT: None declared, M. Le Corvec Employee of: DIAFIR, A. MARTIN: None declared, X. GUENNOC: None declared, C DAVID: None declared, S. HOANG: None declared, C. GUEDES: None declared M. FERREYRA: None declared, E. HOPPE: None declared, B. LEGOFF: None declared, S. JOUSSE-JOULIN: None declared, H. TARIEL Shareholder of: DIAFIR, O. SIRE Consultant for: DIAFIR, A. JOLIVET-GOUGEON: None declared, P GUGGENBUHL: None declared, O. LOREAL Shareholder of: DIAFIR DOI: 10.1136/annrheumdis-2018-eular.6224

\section{FRI0643 AUTOANTIBODY STATUS IS NOT ASSOCIATED WITH EARLY TREATMENT RESPONSE TO FIRST-LINE METHOTREXATE IN PATIENTS WITH EARLY RHEUMATOID ARTHRITIS}

J. S. Dekkers ${ }^{1}$, S. A. Bergstra ${ }^{1}$, A. Chopra ${ }^{2}$, M. Tikly ${ }^{3}$, J. E. Fonseca ${ }^{4}$, K. SalomonEscoto $^{5}$, T. Huizinga ${ }^{6}$, D. van der Woude ${ }^{6} .{ }^{1}$ Rheumatology, LUMC, Leiden, Netherlands, ${ }^{2}$ Rheumatology, Centre For Rheumatic Diseases, Pune, India, ${ }^{3}$ Division Of Rheumatology, University of Witwatersrand, Johannesburg, South Africa, ${ }^{4}$ Rheumatology Research Unit, Lisbon Academic Medical Centre, Lisbon, Portugal, ${ }^{5}$ Department Of Rheumatology, UMass Memorial Medical Center, Massachusetts, United States, ${ }^{6}$ Department Of Rheumatology, LUMC, Leiden, Netherlands

Background: In rheumatoid arthritis (RA), the relationship between autoantibody status and treatment response to methotrexate remains unclear. As methotrexate is the most widely used anti-rheumatic drug in clinical practice, it would be important to know whether the presence of autoantibodies is associated with better treatment response, since patients may benefit from treatment tailored to "autoantibody status".

Objectives: We investigated the relationship between autoantibody status and remission in newly diagnosed RA-patients treated with first-line methotrexate.

Methods: RA-patients initially treated with methotrexate were selected from an international observational database (METEOR). Patients were stratified into autoantibody-positive (rheumatoid factor (RF)- and/or anti-citrullinated-protein antibodies (ACPA)-positive) or -negative (RF- and ACPA-negative). The effect of autoantibody status on the chance of achieving remission within 3 to 6 months was analysed using Cox-proportional hazards regression.

Results: Data from 1826 RA-patients were available for analysis. DAS remission was achieved in $17 \%(318 / 1,826)$. This was similar in autoantibody-positive $(17 \%$ (282/1629)) and -negative patients (18\% (36/197)). Hence, autoantibody positivity was not associated with remission (HR0.89, $95 \% \mathrm{Cl} 0.57 ; 1.38)$. Similar findings were found when stratified for methotrexate monotherapy (HR0.75, 95\%C $0.41 ; 1.37)$ or combination treatment $(\mathrm{HR} 0.76,95 \% \mathrm{Cl} 0.37 ; 1.54)$. Good physical 
function $(H A Q<0.5)$ was achieved in $33 \%(530 / 1590)$ of all patients. Autoantibody-positivity was also not associated with $\mathrm{HAQ}<0.5$ (HR1.05, $95 \% \mathrm{Cl}$ $0.71 ; 1.57)$.

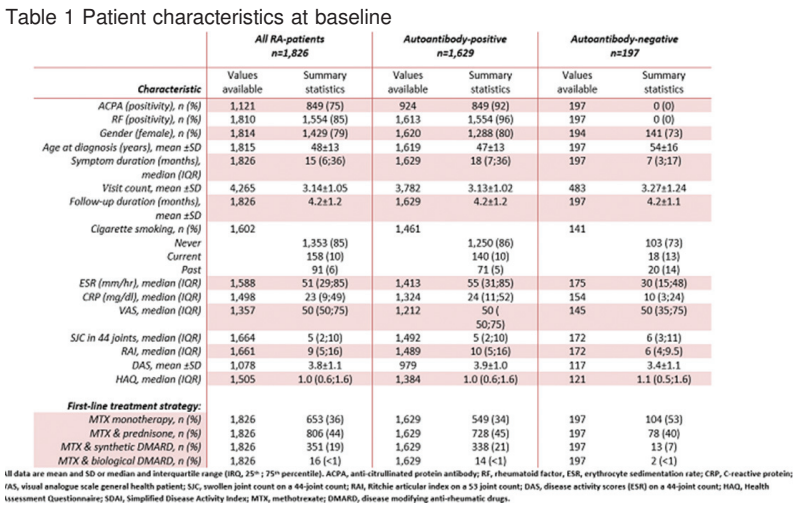

Conclusions: In conclusion, we found that autoantibody status was not associated with early remission in newly diagnosed RA-patients receiving methotrexate in real-world clinical practice. These results do not support the hypothesis that treatment should be tailored to "autoantibody status" when it comes to initiating methotrexate therapy as first-line anti-rheumatic treatment. Rather, our results indicate that that methotrexate is effective as primary anchor drug regardless of autoantibody status.

Disclosure of Interest: None declared

DOI: 10.1136/annrheumdis-2018-eular.4558

\section{FRI0644 THE USE OF MRI-DETECTED SYNOVITIS TO DETERMINE THE NUMBER OF INVOLVED JOINTS FOR THE 2010 ACR/EULAR CLASSIFICATION CRITERIA FOR RHEUMATOID ARTHRITIS - IS IT OF ADDITIONAL BENEFIT?}

A. C. Boer ${ }^{1,{ }^{*}}$, D. Boeters ${ }^{1}$, A. H. M. van der Helm - van Mil ${ }^{1,2} \cdot{ }^{1}$ Rheumatology, Leiden University Medical Center, Leiden, ${ }^{2}$ Rheumatology, Erasmus Medical Center, Rotterdam, Netherlands

Background: The 2010 ACR/EULAR classification criteria have been developed, as early classification of Rheumatoid Arthritis (RA) is important. The 2010criteria states that imaging can be used to determine the number of joints with synovitis. This seems reasonable as previous studies on Magnetic Resonance Imaging (MRI) in early arthritis patients have shown that synovitis is present in a substantial number of joints that were neither swollen nor tender at clinical examination. Although the development of the 2010-criteria was primarily data-driven, the suggestion to also use advanced imaging modalities to detect synovitis was based on expert opinion. Scientific data supporting the use of MRI is lacking.

Objectives: To assess the value of MRI-detected synovitis to determine the number of involved joints on the performance of the 2010-ACR/EULAR classification criteria for RA.

Methods: 277 consecutive patients with a clinical diagnosis of RA or undifferentiated arthritis (UA) were studied. They underwent contrast enhanced 1.5T MRI of MCP-, wrist- and MTP-joints at baseline. Two outcomes were studied after 1 year follow-up: disease modifying anti-rheumatic drugs (DMARD)-initiation and fulfilling the 1987-criteria. Test characteristics were calculated when the number of involved joints was determined with and without MRI-detected synovitis.

Results: At baseline, 143 of 277 patients did not fulfil the 2010-criteria when the number of involved joints was determined by clinical evaluation of swelling and tenderness. When MRI-detected synovitis was also considered 69 patients had increased joint counts. Of these, 36 patients received more points for the item 'number of involved joints' and 14 reached $\geq 6$ points and now fulfilled the 2010criteria for RA. Thus, $10 \%$ of patients that were formally classified as UA were additionally classified as having $R A$.

Without considering MRI-detected synovitis, the sensitivity of the 2010-criteria was $62 \%$ and the specificity $90 \%$, for DMARD initiation as outcome. With the addition of MRI-detected synovitis, the sensitivity increased to $67 \%$ and the specificity decreased to $84 \%$. The AUC changed from 0.76 to 0.75 . The net proportion of correctly reclassified patients was $-2.4 \%$. Of the additionally classified patients, $64 \%$ $(9 / 14)$ were started on DMARDs and were considered true positives, whereas $36 \%(5 / 14)$ were not treated with DMARDs and developed alternative clinical diagnoses during the first year.

Results for the outcome 1987-criteria fulfilment after 1-year were similar. The sensitivity changed from $79 \%$ to $81 \%$ and the specificity from $78 \%$ to $71 \%$ the proportion or correctly reclassified patents was $-5.1 \%$.
Conclusions: To our knowledge, this study is the first providing evidence on the value of MRI-detected synovitis in addition to tender and swollen joints for the classification of RA. We did not find an increased accuracy of the 2010 criteria when incorporating MRI-detected synovitis. Further research on this subject in other longitudinal cohorts is needed, but at present there is no scientific proof that MRI-detected synovitis is of additional benefit for classifying RA.

Disclosure of Interest: None declared DOI: 10.1136/annrheumdis-2018-eular.4501

\section{FRI0645 ANTI-DRUG ANTIBODIES TO CERTOLIZUMAB PEGOL ARE ASSOCIATED WITH LOW DRUG LEVELS AND REDUCED CLINICAL RESPONSE AT 3 MONTHS IN PATIENTS WITH INFLAMMATORY JOINT DISEASES. DATA FROM THE NOR-DMARD STUDY.}

J. E. Gehin ${ }^{1}$, G. L. Goll ${ }^{2}$, D. J. Warren ${ }^{1}$, S. W. Syversen ${ }^{2}$, J. Sexton ${ }^{2}$, E. K. Strand ${ }^{3}$, T. K. Kvien ${ }^{2}$, N. Bolstad ${ }^{1}$, E. Lie ${ }^{2}$. ${ }^{1}$ Department of Medical Biochemistry, Oslo University Hospital, ${ }^{2}$ Department of Rheumatology, Diakonhjemmet Hospital, Oslo, ${ }^{3}$ Lillehammer Hospital for Rheumatic Diseases, Lillehammer, Norway

Background: Anti-drug antibodies (ADAb) to biological drugs predispose patients to low drug levels and lack of treatment response. For certolizumab pego (CP) knowledge about the frequency and clinical relevance of ADAb is limited in patients with inflammatory joint diseases (IJD).

Objectives: To assess the frequency and clinical relevance of early ADAb development in patients with inflammatory joint diseases treated with CP.

Methods: Patients from the NOR-DMARD study $(n=310)$ with a clinical diagnosis of rheumatoid arthritis (RA, $n=91$ ), psoriatic arthritis (PsA, $n=61)$, axial spondyloarthritis (axSpA, $n=116)$ and other IJD (42) starting treatment with $\mathrm{CP}$, who had available biobank sample at 3 months follow-up, were included. Serum samples are non-trough samples collected at 3 months. Drug concentrations were analysed using an in-house immunofluorometric assay automated on the AutoDELFIA immunoassay platform. ADAb was detected by a principal assay measuring neutralising $A D A b$ and two confirmational tests (antigen-bridging test and a 3-step immunofluorometric assay). Patients with RA, PsA and axSpA were included in response analyses. Treatment response was defined by EULAR good/moderate response in RA, DAS28 improvement $>0.6$ in PsA, and ASDAS clinically important improvement (CII) in axSpA.

Results: After 3 months of treatment, 19 of $310(6.1 \%)$ patients were ADAb positive (5 RA, 4 PsA, 6 axSpA and 4 other IJD). ADAb positive patients had significantly lower CP levels than ADAb negative patients, median 1.0 (IQR $0.2-6.8$ ) vs 34.4 (IQR 21.2-44.7) mg/L $(P<0.001)$. Response data were available for 245 patients. Of these, only $1 / 11(9 \%)$ ADAb-positive patients was classified as a res ponder, while 10/11 (91\%) were non-responders. Among ADAb-negative patients with response data, 129/234 (55\%) were responders, while 105/234 (45\%) were non-responders.

Conclusions: ADAb against CP were detected in $6.1 \%$ of patients after 3 months of treatment and were associated with low drug levels and reduced treatment response. These results suggest that drug levels and ADAb may be important for monitoring efficacy of treatment with TNF inhibitors, but the clinical significance needs to be examined in randomised clinical strategy trials.

Disclosure of Interest: J. E. Gehin Consultant for: Roche, G. Goll Consultant for: Abbvie, Biogen, Boehringer Ingelheim, Orion Pharma, Eli Lilly, Novartis, Pfizer, MSD, Roche, UCB, D. Warren: None declared, S. Syversen Consultant for: Roche, J. Sexton: None declared, E. Strand Consultant for: Pfizer, T. Kvien Consultant for: AbbVie, Biogen, BMS, Boehringer Ingelheim, Celgene, Celltrion, Eli Lilly, Epirus, Hospira, Merck-Serono, MSD, Mundipharma, Novartis, Oktal, Orion Pharma, Hospira/Pfizer, Roche, Sandoz, UCB, N. Bolstad Consultant for: Pfizer, Orion Pharma, Napp pharmaceuticals, Takeda, Roche, E. Lie: None declared

DOI: 10.1136/annrheumdis-2018-eular.5257

\section{FRI0646 SYNOVIAL TISSUE HISTOPATHOLOGY FINDINGS IN EARLY RA. IS IT USEFULL? ANALYSIS OF THE BELGIAN CAP48 COHORT.}

C. Galant $^{1}$, L. Meric De Bellefon ${ }^{2}$, B. Lauwerys ${ }^{2}$, T. Sokolova $^{2}$, M. Stoenoiu $^{2}$, V. Badot ${ }^{3}$, A. Nzeusseu', F. Houssiau' ${ }^{2}$, P. Durez ${ }^{2}$. ${ }^{1}$ Pathology, ${ }^{2}$ Rheumatology, Cliniques universitaires Saint-Luc - Université Catholique de Louvain - Institut de Recherche Expérimentale et Clinique (IREC), ${ }^{3}$ Internal Medicine, Centre Hospitalier Universitaire Brugmann, Brussels, Belgium

Background: The development of ultrasound-guided synovial biopsy will enable synovial tissue collection from small joints and will facilitate histopathological studies, thus improving the understanding of early rheumatoid arthritis (ERA). The CAP48 cohort is an original multicentre prospective observational study of early 